Pacific Journal of Mathematics

THE CANONICAL BUNDLE AND REALIZABLE C 


\title{
THE CANONICAL BUNDLE AND REALIZABLE CR HYPERSURFACES
}

\author{
HOWARD JACOBOWITZ
}

\begin{abstract}
The canonical bundle of a realizable $C R$ hypersurface has closed sections. Examples are given of non-realizable hypersurfaces with closed sections and others without such sections. If however an abstract CR hypersurface of dimension $2 m+1$ has $m$ strongly independent CR functions then a closed section can be used to produce the missing function and so assures that the hypersurface is realizable. The existence of a closed section is equivalent to a condition on the range of $\bar{\partial}_{b}$ acting on functions. Some non-realizable CR hypersurfaces are shown to have $\bar{\partial}_{b}$ =cohomology groups quite different from those of realizable hypersurfaces.
\end{abstract}

1. We start with a real manifold $M$ and a sub-bundle $V$ of $\mathbf{C} \otimes T M$. Then $(M, V)$ is called a CR structure (or CR manifold) if $V \cap \bar{V}=\{0\}$ and $[V, V] \subset V$. We will primarily be concerned with $\mathrm{CR}$ structures of hypersurface type; this means $\operatorname{dim}_{\mathbf{R}} M=2 m+1$ and $\operatorname{dim}_{\mathbf{C}} V=m$. Such a $\mathrm{CR}$ structure is realizable if there is an embedding

$$
\phi: M \rightarrow \mathbf{C}^{m+1} \quad \text { with } \phi_{*} V \subset\left\{\frac{\partial}{\partial \bar{z}_{1}}, \ldots, \frac{\partial}{\partial \bar{z}_{m+1}}\right\} .
$$

If $L_{1}, \ldots, L_{m}$ are a basis for $V$ then $(M, V)$ is realizable exactly when the homogeneous equations

$$
L_{j} h=0 \text { for } j=1, \ldots, m
$$

have $m+1$ independent solutions. Not all abstract CR hypersurfaces are realizable $[\mathbf{N 1}, 2],[\mathbf{J T 1}, 2,3]$. It is easy to show that $(M, V)$ is realizable if it admits a one-parameter group of CR diffeomorphisms transverse to $V$ (see below) or if $V$ is a real analytic bundle. There seem to be no other useful characterizations of realizable hypersurfaces. In particular, although the solvability of $L_{j} u=f_{j}$ is well understood there are no similar results for (1) to have non-trivial solutions.

We now wish to define the canonical bundle $K$ of a CR structure (of hypersurface type). Let $\Lambda^{p}$ denote the space of $C^{\infty} p$-forms on $M$ and let $i_{x}: \Lambda^{p} \rightarrow \Lambda^{p-1}$ be inner multiplication with the vector field $X$ (see for instance [S]). For typographical convenience we sometimes use $X\lrcorner$ in 
place of $i_{x}$. The canonical bundle is

$$
K=\left\{\Omega \in \Lambda^{m+1}: i_{L} \Omega=0 \text { for all } L \in V\right\}
$$

It is easy to see that $K$ is a complex line bundle over $M$. Note also that

$$
V=\left\{L \in \mathbf{C} \otimes T M: i_{L} \Omega=0 \text { for all } \Omega \in K\right\} .
$$

As an example consider a realizable CR structure (and identify $M$ with its image in $\mathbf{C}^{m+1}$ ). Then $d z_{1} \wedge \cdots \wedge d z_{m+1}$ restricts to a non-zero form $\Omega$ on $M$ and $\Omega$ generates $K$. Thus in the realizable case $K$ has a closed non-zero section. It is natural to wonder about the converse. See for instance remarks in [F] where the canonical bundle is used to give an interesting construction of the Fefferman metric. Theorem 1 below shows that the canonical bundle of a non-realizable CR manifold can admit a closed non-zero section. (To save words, let us now use "section" to include non-zero.) Note also that every real analytic CR manifold has closed sections of $K$. But it is not true that all canonical bundles have closed sections, (see Corollary 2.1). However, all canonical bundles do share a somewhat weaker property which is a consequence of $[V, V] \subset V$ :

Proposition 1.1. For each section $\Omega$ of $K$ we have some 1 -form $\phi$ such that $d \Omega=\phi \wedge \Omega$.

This property can also be expressed as

$$
d K \subset \mathscr{I}(K)
$$

where $\mathscr{I}(K)$ is the ideal generated by $K$. Note that if $\Omega$ is any section and if $\Omega_{1}$ is a closed section then setting $\Omega=\lambda \Omega_{1}$ for some non-zero function $\lambda$, we have

$$
d \Omega=d \lambda \wedge \Omega_{1}=\phi \wedge \Omega
$$

and so (2) is a weaker property than having a closed section.

A class of non-realizable CR manifolds was given by LeBrun [LeB] using ideas related to the Penrose twistor program. It is easy to verify that the canonical bundle of each of these manifolds admits closed sections. We do this in a slightly different context. Let $f(x, \zeta)$ be a function on $\mathbf{R}^{3} \times \mathbf{C}^{3}$ which is holomorphic in $\zeta$. (We work locally so we actually mean $f$ is holomorphic near some distinguished point.) We take $f$ holomorphic in order to simplify the presentation; the construction would also work for suitable non-holomorphic functions. One could replace $\mathbf{R}^{3} \times \mathbf{C}$ by $\mathbf{R}^{n} \times \mathbf{C}^{n}$ but then the CR manifold is no longer of hypersurface type, cf. [R]. 
THEOREM 1. Assume that at some point $p$ the vector $\left(f_{\zeta_{1}}, f_{\zeta_{2}}, f_{\zeta_{3}}\right)$ is not a multiple of a real vector. Then near $p, N=\{(x, \zeta): f(x, \zeta)=0\}$ can be given a CR structure which has a closed section of the canonical bundle. However for some choice of $f$ and $p$ this structure is non-realizable.

Let $x$ and $\zeta$ be the usual coordinates on $\mathbf{R}^{3} \times \mathbf{C}^{3}$ and let $\omega$ be the restriction to $N$ of the 2 -form $d x d \zeta=\sum_{j=1}^{3} d x_{j} \wedge d \zeta_{j}$. Let $V=\{L \in \mathbf{C}$ $\left.\otimes T N: i_{L} \omega=0\right\}$. To show that $V$ gives a CR structure (of hypersurface type) we need to show

$$
\begin{gathered}
\operatorname{dim}_{\mathbf{C}} V=3 \\
V \cap \bar{V}=\{0\} \\
{[V, V] \subset V .}
\end{gathered}
$$

So let

$$
L=\sum_{1}^{3} \alpha_{j} \frac{2}{\partial \zeta_{j}}+\beta_{j} \frac{2}{\partial \bar{\zeta}_{j}}+\gamma_{j} \frac{2}{\partial x_{j}} .
$$

The condition $i_{L} \omega=0$ is the same as $i_{L} d x d \zeta=A d f+B d \bar{f}$. But since $f$ is holomorphic (and $d_{\zeta} f \neq 0$ ) we must have that $B=0$. Thus each $\alpha_{j}$ and $\gamma_{j}$ is determined up to the complex parameter $A$. Further since $i_{L} i_{L} d x d \zeta$ must be zero, we see that $L(f)=0$. The condition $L(\bar{f})=0$ then allows us to eliminate one $\beta$. Thus $\left\{L \in \mathbf{C} \otimes T\left(R^{3} \times \mathbf{C}^{3}\right): L(f)=L(\bar{f})=0\right.$ and $\left.i_{L} \omega=0\right\}$ has dimension three and this set clearly is the set $V$. So (a) is verified.

Note that in the above $\gamma_{j}=A\left(\partial f / \partial \zeta_{j}\right)$. Thus for any non-zero $A$ the vector $\left(\gamma_{1}, \gamma_{2}, \gamma_{3}\right)$, and so also the vector field $L$, cannot be real. And if $A$ is zero then also each $\alpha_{j}$ is zero and $L$ can be real only if $\beta_{j}$ is also zero, i.e. only if $L=0$. This verifies (b).

Now note that if $\left.L_{1}\right\lrcorner \omega=0$ and $\left.L_{2}\right\lrcorner \omega=0$ and if $U$ is any vector in $\mathbf{C} \otimes T N$ then $d \omega\left(L_{1}, L_{2}, U\right)=-\omega\left(\left[L_{1}, L_{2}\right], U\right)$. But $\omega$ is closed, thus $\left.\left[L_{1}, L_{2}\right]\right\lrcorner \omega$ must also be zero. This verifies (c). It should be pointed out that whenever $\omega$ is a real closed form of any degree on some manifold $M$ the real bundle $V=\left\{L \in T M, i_{L} \omega=0\right\}$ satisfies $[V, V] \subset V$ and so defines integral submanifolds. Of course in our case $V$ is complex and so $[V, V] \subset V$ does not imply the existence of integral submanifolds.

Now let $\Omega=\omega \wedge \omega \in \Lambda^{4}$. It is easy to see that $\Omega$ is a nowhere zero form. In fact if $\partial f / \partial \zeta_{3} \neq 0$ then $\left(x_{1}, x_{2}, x_{3}, \zeta_{1}, \zeta_{2}\right.$ may be taken as coordinates for $N$ and

$$
\Omega\left(\frac{\partial}{\partial x_{1}}, \frac{\partial}{\partial x_{2}}, \frac{\partial}{\partial \zeta_{1}}, \frac{\partial}{\partial \zeta_{2}}\right) \neq 0
$$


Also $i_{L} \Omega=\left(i_{L} \omega\right) \wedge \omega+\omega \wedge\left(i_{L} \omega\right)=0$ since $i_{L} \omega=0$. Thus $\Omega$ is a section of $K$. And $d \Omega=0$ since $d \omega=0$.

Finally, note that the hypothesis of Proposition 1.2 is satisfied away from the point $\zeta=0$ by any function $f(x, \zeta)=g_{i j}(x) \zeta_{i} \zeta_{j}$ when the matrix $g$ is real and positive definite. Choose $g$ to equal the identity matrix $I$ to infinite order at $x=0$ but $g$ to be not conformally equivalent to $I$ as germs at $x=0$. Then there is no real analytic metric in the conformal class of $g$. We now use [LeB] to show this implies $N$ is not locally realizable. Note that the fibres of $N$ over points in $\mathbf{R}^{3}$ are complex surfaces. This implies that the Levi form of $N^{7}$ has a zero eigenvalue. We first find a quotient manifold $N^{5}$ with signature $(1,1)$. (It is useful to say a matrix with $p$ positive and $q$ negative eigenvalues, and no zero eigenvalues, has signature $(p, q)$ rather than $p-q$.) So let $\mathbf{C}^{*}=\mathbf{C}-\{0\}$ act on the fibres of $N^{7}-\{0$ section $\}$. It is easy to see that the quotient manifold $N^{5}$ is also a CR manifold and has $\mathbf{C P}^{1}$ as fibre. A calculation shows $N^{5}$ has signature $(1,1)$. Le Brun, op cit, uses the $\mathbf{C P}^{1}$ foliation to show that $N^{5}$ cannot be locally realizable in the neighborhood of each of its points. We now need only verify that this implies $N^{7}$ is also somewhere non-realizable. Let $p$ be some point in the fibre above $0 \in \mathbf{R}^{3}$ and let $[p]$ be the corresponding point in $N^{5}$. We claim that if $N^{7}$ is realizable at $p$ then $N^{5}$ is realizable at $[p]$. Thus we assume $N^{7}$ is given by a real hypersurface in $\mathrm{C}^{4}$ and $p$ is the origin. Let $X \in V$ with $\pi_{*} X=0$, where $\pi: N^{7} \rightarrow N^{5}$. We may assume $X=\partial / \partial z_{4}$. Then $\left\{z \in \mathbf{C}^{4}: z_{4}=0\right\} \cap N^{7}=M^{5}$ is a real hypersurface in $\mathbf{C}^{3}$.

The complex curve $\pi^{-1}[0]$ is transverse to $\mathbf{C}^{3}=\left\{z_{4}=0\right\}$. So for $[p]$ close to $[o]$ the complex curve $\pi^{-1}[p]$ is also transverse to $\mathbf{C}^{3}$ and thus intersects $\mathbf{C}^{3}$ in a single point. Thus the map $\pi: N^{7} \rightarrow N^{5}$ when restricted to $M^{5} \subset N^{7}$ gives a CR diffeomorphism (see $\S 2$ for definition) of $M^{5}$ to $N^{5}$. The inverse of this map gives a CR realizable of $N^{5}$ as a hypersurface in $\mathrm{C}^{3}$. But $N^{5}$ is not locally realizable; thus there must be some $p$ at which $N^{7}$ is not realizable. This concludes the proof of Theorem 1. It is not clear whether $N^{5}$ also has a closed section of its canonical bundle.

Thus the existence of a closed section of $K$ cannot by itself imply realizability. It is natural to wonder if it suffices to add an assumption about the signature of the Levi form. Note that strictly pseudo-convex hypersurfaces of dimension greater than 7 are always realizable [K] and so have closed sections.

Question: Let $\left(M^{2 m+1}, V\right)$ have signature $(p, m-p)$ with $p \neq 1$ or $m-1$. Must $K$ have a closed section? 
As we have just indicated, the answer is "yes" when $p=0$ or $m$ as long as $m \geq 4$. A positive answer in the other cases could be viewed as a weak realizability result. It is natural to exclude $p=1$ and $p=m-1$ since in these cases $\bar{\partial}_{b}$, for realizable hypersurfaces, is not solvable on $(0,1)$-forms. A better reason for excluding this case would follow if our counterexample for $N^{7}$ could be extended to $N^{5}$. See also the remark after Theorem 4.

2: We will study the realization problem and its relation to closed sections of $K$ using a complex vector field formally analogous to the generator of a local one-parameter group of CR diffeomorphisms.

For a real vector field $X$ let $\mathscr{L}_{X}$ denote the Lie derivative acting on forms, vector fields, etc. (see for example [S] for the definition and basic properties). Recall the identity

$$
\mathscr{L}_{X} \omega=d\left(i_{X} \omega\right)+i_{X}(d \omega)
$$

where $\omega$ is any differential form. If $Y=X_{1}+i X_{2}$ is a complex vector field we write $\mathscr{L}_{Y}$ to mean the operator $\mathscr{L}_{X_{1}}+i \mathscr{L}_{X_{2}}$. Then (3) is also valid for $Y$ in place of $X$.

Let $\psi: M \rightarrow M$ be a diffeomorphism (of a neighborhood of some point $p$ to a neighborhood of some point $q$ ). It is called a CR diffeomorphism if $\psi_{*} V=V$. Now let $\phi(t): M \rightarrow M$ be a local one-parameter group of CR diffeomorphisms and let $Y=(\partial / \partial t)$ be the real vector field which is its generator. It follows from the definition of $\mathscr{L}$ that $\mathscr{L}_{Y} V \subset V$. Conversely given a real vector field $Y$ with $\mathscr{L}_{Y} V \subset V$ then the group of diffeomorphisms generated by $Y$ preserves the CR structure. As the complex analogue of this real generator we will consider complex vector fields satisfying $\mathscr{L}_{Y} V \subset V$. There are always such vector fields: If $L$ is a section of $V$ and $P$ is any other section of $V$ then $\mathscr{L}_{L} P=[L, P] \subset V$, i.e. $\mathscr{L}_{L} V \subset V$. We soon shall see that $(M, V)$ is realizable precisely when there is a vector field transverse to $V \oplus \bar{V}$ which also satisfies $\mathscr{L}_{Y} V \subset V$.

LEMMA 2.1. For any vector field $Y$ the following are equivalent:

(a) $\mathscr{L}_{Y} V \subset V$.

(b) $\mathscr{L}_{Y} K \subset K$.

(c) For every section $\Omega$ of $K$ there is some function $\lambda$ such that $\mathscr{L}_{Y} \Omega=\lambda \Omega$.

(d) There is some section $\Omega$ of $K$ and some function $\lambda$ such that $\mathscr{L}_{Y} \Omega=\lambda \Omega$.

Proof. Since $K$ is one dimensional (b) implies (c). And certainly (c) implies (d). So we need only prove that (a) implies (b) and (d) implies (a). 
Let $L$ be a section of $V$ and $\Omega$ a section of $K$. From the identity

$$
\left.\left.\left.\mathscr{L}_{Y}(L\lrcorner \Omega\right)=\left(\mathscr{L}_{Y} L\right)\right\lrcorner \Omega+L\right\lrcorner \mathscr{L}_{Y} \Omega
$$

we see that

$$
\left.\left.\left(\mathscr{L}_{Y} L\right)\right\lrcorner \Omega+L\right\lrcorner \mathscr{L}_{Y} \Omega=0 .
$$

Both the desired implications follow from this equation.

In particular note that for any sections $L$ and $\Omega$ of $V$ and $K$ we have

$$
\mathscr{L}_{L} \Omega=\lambda \Omega .
$$

It is well known that if a CR manifold admits a one parameter group of $\mathrm{CR}$ diffeomorphisms then that $\mathrm{CR}$ manifold is realizable. We give a very simple proof of this below. Of course most $\mathrm{CR}$ manifolds, realizable or not, do not admit such diffeomorphisms.

Thus the next result is somewhat surprising.

Proposition 2.1. The following are equivalent:

(a) $(M, V)$ is realizable in a neighborhood of the point $p$.

(b) There exists a vector field $Y$ with $\mathscr{L}_{Y} V \subset V$ and $Y \notin V \oplus \bar{V}$ at $p$.

Proof of $\mathrm{a} \Rightarrow \mathrm{b}$. We may assume $M^{2 m+1} \subset \mathbf{C}^{m+1}$ with $V$ at $p$ given by $\left\{\partial / \partial \bar{z}_{1}, \ldots, \partial / \partial \bar{z}_{m}\right\}$. Necessarily, near $p, d z_{1} \wedge \cdots \wedge d z_{m} \wedge d \bar{z}_{1}$ $\wedge \cdots \wedge d \bar{z}_{m} \wedge d z_{m+1}$ is a non-zero form on $M$. This has two consequences for us. First, there is a unique vector field on $M$ satisfying

$$
d z_{m+1}(Y)=1 \quad \text { and } \quad d z_{j}(Y)=0=d \bar{z}_{j}(Y) \text { for } j=1, \ldots, m .
$$

We claim this field is transverse to $V \oplus \bar{V}$. On the contrary, assume that at some point $Y=Y_{1}+Y_{2}$ with $Y_{1} \in V$ and $Y_{2} \in \bar{V}$. Then $d \bar{z}_{j}\left(Y_{1}\right)=0$ for $j=1, \ldots, m$. But since $Y_{1} \in V$, this implies $Y_{1}=0$. Similarly $d z_{j}\left(Y_{2}\right)=0$ and so $Y_{2} \in \bar{V}$ implies $Y_{2}=0$. Here we used that also $d z_{1} \cdots d z_{m} d \bar{z}_{1} \cdots$ $d \bar{z}_{m} d \bar{z}_{m+1}$ is non-zero.

Second, the form $\Omega=d z_{1} d z_{2} \cdots d z_{m+1}$ is non-zero and hence gives a section of $K$. Note that both $\Omega$ and $i_{Y} \Omega$ are closed. Hence according to (3), $\mathscr{L}_{Y} \Omega=0$. But then, by Lemma 2.1, $\mathscr{L}_{Y} V \subset V$.

Proof of $\mathrm{b} \Rightarrow \mathrm{a}$. Let $t$ be the coordinate on $R$ and let $V_{1} \subset \mathbf{C} \otimes$ $T(R \times M)$ be the subpace obtained by extending each vector of $V$ to be independent of $t$. Similarly extend $Y$ and then take $Z=Y+i(\partial / \partial t)$. We may assume that $\operatorname{Re} Y \notin V \oplus \bar{V}$ at $p$. Thus at $p, W=V_{1} \oplus\{\alpha Z: \alpha \in \mathbf{C}\}$ satisfies $W \cap \bar{W}=\{0\}$ and so gives an almost complex structure on $R \times M$. From $\mathscr{L}_{Y} V \subset V$ and the fact that all extensions are independent of $t$, we see that this structure is integrable, i.e. $[W, W] \subset W$. Thus by the 
Newlander-Nirenberg theorem ([NN], see also [FK] for this formulation) $W$ gives a complex structure. The submanifold $\{0\} \times M$ realizes the $\mathrm{CR}$ structure $(M, V)$ as a hypersurface in $\mathrm{C}^{m+1}$.

We have seen that $K$ may have a closed section without $(M, V)$ being realizable. However if we already have $m$ of the required $m+1$ functions (and they are suitably general) then we can use a closed section to construct the missing function (Theorem 2, below). Recall that a function $f$ is a CR function for $(M, V)$ if $L f=0$ for each $L \in V$. It is easy to show that an embedding $M \rightarrow \mathbf{C}^{m+1}$ given by functions $\phi_{1}, \ldots, \phi_{m+1}$ realizes $(M, V)$ as a hypersurface in $\mathrm{C}^{m+1}$ if each $\phi_{j}$ is a $\mathrm{CR}$ function. Recall also that $\phi_{1}, \ldots, \phi_{n}$ are independent at a point $p$ if $d \phi_{1} \wedge \cdots \wedge d \phi_{n} \neq 0$ at $p$. Let us say they are "strongly independent" there if also $d \phi_{1} \wedge \cdots \wedge d \phi_{n}$ $\wedge d \bar{\phi}_{1} \wedge \cdots \wedge d \bar{\phi}_{n} \neq 0$. As an example consider the Lewy operator $L=$ $\partial / \partial \bar{z}-i z(\partial / \partial u)$ and the two solutions $\phi=z$ and $\psi=u+i|z|^{2}$. Then $d \phi$ and $d \psi$ are each non-zero so each is an independent function at the origin.

However $\phi$ is also strongly independent at the origin while $\psi$ is not. Note, as an illustration of Lemma 2.4 , then $d \phi \wedge d \bar{\phi} \wedge d \psi \neq 0$. It is possible for a CR structure to have an independent solution (i.e. $d \phi \neq 0$ ) but no strongly independent solution (i.e. $d \phi \wedge d \bar{\phi}=0$ for all solutions). This is easily seen using the technique introduced in [JT1]. Specifically one can find a perturbation function $f(z, \bar{z}, u)$ such that for the operator

$$
L=\frac{\partial}{\partial \bar{z}}-i z \frac{\partial}{\partial u}+f\left(\frac{\partial}{\partial z}-i \bar{z} \frac{\partial}{\partial u}\right)
$$

one has $L h=0$ implies $d h \wedge d u=0$ at the origin. Thus $u+i|z|^{2}$ is an independent solution and there are no strongly independent solutions.

LEMMA 2.3. If $\left\{\phi_{1}, \ldots, \phi_{m}\right\}$ is a strongly independent set of $C R$ functions for $\left(M^{2 m+1}, V\right)$ then $d \phi_{1} \wedge \cdots \wedge d \phi_{m}$ is a non-zero form on $\bar{V}$.

Proof. Let $L_{1}, \ldots, L_{m}$ be a basis for $V$. Write $d \phi$ for $d \phi_{1} \wedge \cdots \wedge d \phi_{m}$ and $L$ for $\left(L_{1}, \ldots, L_{m}\right)$.

Let $L, \bar{L}$, and $X$ be a basis for $\mathbf{C} \otimes T M$ at the given point with $d \phi_{j}(X)=0=d \bar{\phi}_{j}(X)$ for each $j$. Let $Y=\left(Y_{1}, \ldots, Y_{2 m}\right)$ be a choice of $2 m$ vectors from the set $\{L, \bar{L}, X\}$ of $2 m+1$ vectors. Then $d \phi \wedge d \bar{\phi}(Y)$ $=0$ if $X$ appears in $Y$. So $d \phi \wedge d \bar{\phi} \neq 0$ implies $d \phi \wedge d \bar{\phi}(L, \bar{L}) \neq 0$. But $d \phi \wedge d \bar{\phi}(L, \bar{L})=(-1)^{m+1}|d \phi(\bar{L})|^{2}$ and thus $d \phi$ is non-zero on $\bar{V}$.

When $(M, V)$ is realizable, strongly independent $\mathrm{CR}$ functions can be take for coordinate functions as shown in our next proof. This lemma is a standard result which already was pointed out by Lewy [L]. 
LEMMA 2.4. If $\left(M^{2 m+1}, V\right)$ has $m+1$ independent $C R$ functions then $(M, V)$ is realizable.

Proof. We work at some fixed point $p$. We label the CR functions so that at $p$

$$
d \bar{\phi}_{m+1} \in\left\{d \phi_{1}, \ldots, d \phi_{m+1}, d \bar{\phi}_{1}, \ldots, d \bar{\phi}_{m}\right\} .
$$

We claim that then

$$
d \phi_{1} \wedge \cdots \wedge d \phi_{m+1} \wedge d \bar{\phi}_{1} \wedge \cdots \wedge d \bar{\phi}_{m} \neq 0 .
$$

For if it is equal to zero at $p$ then after relabelling we have

$$
d \bar{\phi}_{m} \in\left\{d \phi_{1}, \ldots, d \phi_{m+1}, d \bar{\phi}_{1}, \ldots, d \bar{\phi}_{m-1}\right\} \equiv W
$$

and so $d \bar{\phi}_{m+1}$ also is an element of $W$. Note $\operatorname{dim}_{C} W \leq 2 m$. Hence there is some non-zero vector $X$ annihilated by $W$ and so also by $d \bar{\phi}_{m}$ and $d \bar{\phi}_{m+1}$. The hypothesis then assures $X \in V \cap \bar{V}$ which contradicts $X \neq 0$. This gives (5). Thus the functions $\operatorname{Re} \phi_{1}, \operatorname{Im} \phi_{1}, \ldots, \operatorname{Re} \phi_{m+1}, \operatorname{Im} \phi_{m+1}$ provide an embedding of $M^{2 m+1}$ into $\mathbf{R}^{2 m+2}$. It is easy to see that $(M, V)$ is realized by this as a hypersurface in $\mathbf{C}^{m+1}$. Note that $\left(\phi_{1}, \ldots, \phi_{m}\right)$ is strongly independent and agrees with the restrictions of $\left\{z_{1}, \ldots, z_{m}\right\}$ to $M \subset \mathbf{C}^{m+1}$.

THEOREM 2. If near some point $p\left(M^{2 m+1}, V\right)$ has $m$ strongly independent $C R$ functions and its canonical bundle has a closed section then $\left(M^{2 m+1}, V\right)$ is realizable on some neighborhood of $p$.

Proof. We need only modify some previous arguments. We first show that under these hypotheses there is some vector field $Y$ such that $d\left(i_{Y} \Omega\right)=0$ where $\Omega$ is the closed section of $K$. To see this let $\phi_{1}, \ldots, \phi_{m}$ be strongly independent CR functions. Let $\theta$ be any non-zero one-form which annihilates $V \oplus \bar{V}$. Then $\theta \wedge d \phi \subset K$. (Again we use $d \phi=d \phi_{1}$ $\wedge \cdots \wedge d \phi_{m}$ and let $L=L_{1}, \ldots, L_{m}$ be a basis for $V$.) If $X$ is transverse to $V \oplus \bar{V}$ then

$$
\theta \wedge d \phi(X, \bar{L})=\theta(X) d \phi(\bar{L}) \neq 0
$$

and

$$
\theta \wedge d \phi \wedge d \bar{\phi}(X, \bar{L}, L)=\theta(X)|d \phi(\bar{L})|^{2} \neq 0 .
$$

In particular $\theta \wedge d \phi$ is a non-zero form and so gives a section of $K$. Now pick some closed section $\Omega$ of $K$. We have $\Omega=f \theta \wedge d \phi$ for some 
non-zero function $f$. Define $Y$ by

$$
\theta(Y)=1 / f, \quad d \phi_{j}(Y)=0, \quad d \bar{\phi}_{j}(Y)=0, \quad j=1, \ldots, m .
$$

Certainly $Y \notin V \oplus \bar{V}$. And

$$
d\left(i_{Y} \Omega\right)=d(d \phi)=0 .
$$

But since $d \Omega=0$ we then have $\mathscr{L}_{Y} \Omega=0$ and, by Lemma 2.1 and Proposition 2.1, $(M, V)$ is realizable.

Corollary 2.1. Not all canonical bundles admit closed sections.

Proof. The first example of a non-realizable $\left(M^{3}, V\right)$ (see [N1, Thm. $3^{\prime}$ ) has a strongly independent CR function $z=x+i y$. Thus its canonical bundle cannot have a closed section.

3. In this section we relate the existence of closed sections of $K$ and the range of $\bar{\partial}_{b}$. We conclude with some remarks about the $\bar{\partial}_{b}$-cohomoloby groups. Consider first the case of $\left(M^{3}, V\right)$. Choose any section $L$ of $V$ and any section $\Omega$ of $K$. Consider the function $\lambda$ defined by $\mathscr{L}_{L} \Omega=\lambda \Omega$ (see equation (4)).

THEOREM 3. $K$ has a closed section if and only if there exists a function $f$ with $L(f)=\lambda$.

Proof. Apply (3) with $\omega=g \Omega$. Since $d(g \Omega)$ is a form of top degree we see that $d(g \Omega)=0$ if and only if $\mathscr{L}_{L}(g \Omega)=0$. If $g$ is non-zero then we write $g=e^{-f}$ and we have $\mathscr{L}_{L}(g \Omega)=-g(L f-\lambda) \Omega$. Thus $g \Omega$ is a closed section of $K$ if and only if $L f=\lambda$. (One could give this proof without using $\mathscr{L}$ by simply relating $d$ to $L$. See the proof of Theorem 4.)

We now look more closely at a special case of this theorem. Consider the CR structure given by the operator

$$
L=\frac{\partial}{\partial \bar{z}}-i G(z, \bar{z}, u) \frac{\partial}{\partial u} .
$$

The function $z$ is a strongly independent solution, so there is a second solution $\phi$ with $d \phi \wedge d z \neq 0$ if and only if $K$ has a closed section. Now, $\Omega=(d u+i G d \bar{z}) \wedge d z$ is a section of $K$ and $\mathscr{L}_{L} \Omega=i_{L} d \Omega=-i G_{u} \Omega$. So the solution $\phi$ exists in a neighborhood of the origin (and hence $L$ is realizable) if and only if $L f=G_{u}(z, \bar{z}, u)$ has a solution near the origin. Thus we would have necessary and sufficient conditions for solving 
$L \phi=0$ if we had such conditions for solving $L f=g$. Unfortunately such conditions are only known ([GKS], $[\mathbf{H}])$ when $L$ is realizable, that is when one assumes the existence of $\phi$. But a variation of this can be used to construct simple non-realizable $\mathrm{CR}$ hypersurfaces [J].

Of course, when $L$ is realizable one might expect that $L f=G_{u}$ has an explicit solution. This is indeed the case. For upon differentiating $\phi_{\bar{z}}$ $-i G \phi_{u}=0$ with respect to $u$ we obtain $\left(\phi_{u}\right)_{\bar{z}}-i G\left(\phi_{u}\right)_{u}-i G_{u} \phi_{u}=0$ and so $L\left(\ln \phi_{u}\right)=i G_{u}$. Note that $L \phi=0$ and $d \phi \wedge d z \neq 0$ imply $\phi_{u}(0) \neq 0$ so we indeed have a well-defined solution.

To formulate similar results for $\left(M^{2 m+1}, V\right), m>1$, we use the partial differential operator $\bar{\partial}_{b}$ acting on forms of type $(p, q)$. See for instance $[\mathbf{F K}]$ for a definition of this operator. We use the notation that each $r$-form $\phi \in \Lambda^{r}$ defines an equivalence class $[\phi] \in \sum_{p+q=r} \mathscr{B}^{p, q}$ and $\bar{\partial}_{b}$ maps $\mathscr{B}^{p, q}$ into $\mathscr{B}^{p, q+1}$. Associated to $\bar{\partial}_{b}$ are the cohomology groups $H^{p, q}$ involving germs of forms near a given point $p$. When $(M, V)$ is realizable these groups have the following properties:

$$
H^{0,0} \text { is infinite dimensional }
$$

$$
H^{r, q} \cong H^{s, q}
$$

Neither of these properties need hold for non-realizable $(M, V)$. For $H^{0,0}=$ \{germs at $p$ of CR functions $\}$ and thus if the only CR functions are the constants (as in [JT2 and 3] and [N2]) then $H^{0,0}$ is only one dimensional. Also if $\left(M^{3}, V\right)$ has $z=x+i y$ as a CR function but no other CR function independent of $z$, then $K$ has no closed sections and so $H^{0,0} \neq\{0\}$ but $H^{2,0}=\{0\}$.

Let $\Omega_{1}$ and $\Omega_{2}$ be sections of $K$ and let $d \Omega_{1}=\phi_{1} \wedge \Omega_{1}, d \Omega_{2}=\phi_{2} \wedge$ $\Omega_{2}$ (cf. Prop. 1.1). Note that $\left[\phi_{j}\right] \in \mathscr{B}^{0,1}$ is unique although $\phi_{j}$ is not. Also $\left[\phi_{1}\right]$ is in the range of $\bar{\partial}_{b}$ if and only if $\left[\phi_{2}\right]$ is in this range. This is because $\Omega_{2}=f \Omega_{1}$ and hence $d \Omega_{2}=\left(\bar{\partial}_{b}(\ln f)+\phi_{1}\right) \wedge \Omega_{2}$. So our next result is actually a statement about $K$ rather than any particular section.

THEOREM 4. Let $\left(M^{2 m+1}, V\right)$ be $C R$ structure and let $d \Omega=\phi \wedge \Omega$. Then $K$ has a closed section if and only if $[\phi]$ is in the range of $\bar{\partial}_{b}$ acting on functions.

Proof. Since $[\Omega]$ is a form of type $(m+1,0)$ we have, for any function $g,[(d g) \wedge \Omega]=\left(\bar{\partial}_{b} g\right) \wedge[\Omega]$. So if $d \Omega=\phi \wedge \Omega$ then for any non-zero function $[d(g \Omega)]=g\left(\bar{\partial}_{b} \ln g+[\phi]\right) \wedge[\Omega]$. The theorem now follows from the observation that for $\Omega_{1} \in \mathscr{B}^{m+1,0}, d\left(\Omega_{1}\right)$ is zero if and only if $\left[d \Omega_{1}\right]$ is zero. 
REMARK. It follows from this theorem that whenever $K$ does not have a closed section then $H^{0,1} \neq\{0\}$. (To see this one need only check that $\bar{\partial}_{b}[\phi]=0$ and thus $[\phi]$ defines a cohomology class.)

\section{REFERENCES}

[F] F. Farris, An intrinsic Construction of Fefferman's CR metric, to appear.

[FK] G. Folland and J. Kohn, The Neumann Problem for the Cauchy-Riemann Complex, Princeton University Press, Princeton, 1972.

[GKS] P. Greiner, J. Kohn and E. Stein, Necessary and sufficient conditions for the solvability of the Lewy equation, Proc. Nat. Acad. Sci., 72 (1975), 3287-3289.

[H] G. Henkin, The equation of $H$. Lewy and analysis on a pseudoconvex manifold, Russian Math. Surveys, 32:3 (1977), 59-130.

[J] H. Jacobowitz, A simple example of a non-realizable hypersurface, to appear Proc. Amer. Math. Soc.

[JT1] H. Jacobowitz and F. Treves, Non-realizable CR structures, Invent. Math., 66 (1982), 231-249.

[JT2] Nowhere solvable homogeneous partial differential equations, Bull. Amer. Math. Soc., 8 (1983), 467-470.

[JT3] _ _ Aberrant CR structures, Hokkaido Math. J., 12:1 (1983), 276-292.

[K] M. Kuranishi, Strongly pseudoconvex CR structures over small balls. Part III. An embedding theorem, Annals. of Math., 116 (1982), 249-330.

[leB] C. LeBrun, Twistor CR manifolds and three-dimensional conformal geometry, Trans. Amer. Math. Soc., 284 (1984), 601-616.

[L] H. Lewy, On the local character of the solutions..., Annals of Math., 64 (1956), 514-522.

[NN] A. Newlander and L. Nirenberg, Complex analytic coordinates in almost-complex manifolds, Annals of Math., 65 (1957), 391-404.

[N1] L. Nirenberg, Lectures on linear partial differential equations, Regional Conference Series in Mathematics, Amer. Math. Soc., Providence, 1973.

[N2] _ On a question of Hans Lewy, Russian Math. Surveys, 29 (1974), 251-262.

[R] H. Rossi, LeBrun's nonrealizability theorem in higher dimensions, Duke Math. J., 52 (1985), 457-474.

[S] S. Sternberg, Lectures on Differential Geometry, Second Edition, Chelsea Publishing Co., New York, 1984.

Received September 26, 1985. This work was partially supported by NSF grant DMS8402631 and was done while the author was a Member of the Institute for Advanced Study.

RUTGERS UNIVERSITY

CAMDEN, NJ 08102 



\section{PACIFIC JOURNAL OF MATHEMATICS EDITORS}

\author{
V. S. VARADARAJAN \\ (Managing Editor) \\ University of California \\ Los Angeles, CA 90024 \\ Herbert Clemens \\ University of Utah \\ Salt Lake City, UT 84112 \\ R. FINN \\ Stanford University \\ Stanford, CA 94305
}

\author{
HERMANN FLASCHKA \\ University of Arizona \\ Tucson, AZ 85721 \\ RAMESh A. GANGOLli \\ University of Washington \\ Seattle, WA 98195 \\ VAUGHAN F. R. JONES \\ University of California \\ Berkeley, CA 94720 \\ ROBION KIRBY \\ University of California \\ Berkeley, CA 94720 \\ C. C. MOORE \\ University of California \\ Berkeley, CA 94720 \\ H. SAMELSON \\ Stanford University \\ Stanford, CA 94305 \\ HAROLD STARK \\ University of California, San Diego \\ La Jolla, CA 92093
}

\section{ASSOCIATE EDITORS}
R. ARENS
E. F. BECKENBACH
B. H. NEUMANN
F. WOLF
K. YOSHIDA (1906-1982)

\section{SUPPORTING INSTITUTIONS}
UNIVERSITY OF ARIZONA
UNIVERSITY OF BRITISH COLUMBIA
CALIFORNIA INSTITUTE OF TECHNOLOGY
UNIVERSITY OF CALIFORNIA
MONTANA STATE UNIVERSITY
UNIVERSITY OF NEVADA, RENO
NEW MEXICO STATE UNIVERSITY
OREGON STATE UNIVERSITY
UNIVERSITY OF OREGON UNIVERSITY OF SOUTHERN CALIFORNIA STANFORD UNIVERSITY UNIVERSITY OF HAWAII UNIVERSITY OF TOKYO UNIVERSITY OF UTAH WASHINGTON STATE UNIVERSITY UNIVERSITY OF WASHINGTON 


\section{Pacific Journal of Mathematics \\ Vol. 127, No. $1 \quad$ January, 1987}

Jacob Burbea, Boundary behavior of holomorphic functions in the ball ..... 1

Jan Dijkstra, Strong negligibility of $\sigma$-compacta does not characterize

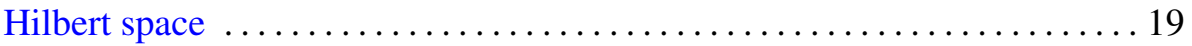

Ruy Exel, Rotation numbers for automorphisms of $C^{*}$ algebras $\ldots \ldots \ldots 31$

Howard Jacobowitz, The canonical bundle and realizable CR

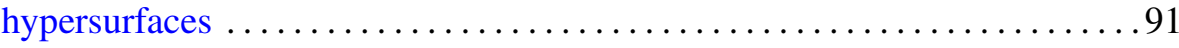

James T. Joichi and Dennis Warren Stanton, Bijective proofs of basic

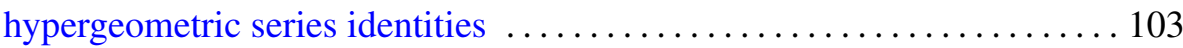

Gareth J. Knowles, Quotients of nest algebras with trivial commutator .... 121

Murray Angus Marshall, Exponentials and logarithms on Witt rings ..... 127

Courtney Hughes Moen, The dual pair $(U(3), U(1))$ over a $p$-adic field . ...141

William Ortmeyer, Surgery on a class of pretzel knots $\ldots \ldots \ldots \ldots \ldots \ldots 155$

John Gerard Ryan, Extensions of representations of Lie algebras . . . . . 173

Ivan Charles Sterling, A generalization of a theorem of Delaunay to

rotational $W$-hypersurfaces of $\sigma_{l}$-type in $H^{n+1}$ and $S^{n+1} \ldots \ldots \ldots 187$

Vesko M. Valov, Another characterization of AE(0)-spaces $\ldots \ldots \ldots \ldots \ldots 9$ 